\title{
Correlation of Different Parameters for Canopy Performance
}

\author{
S. M. Jahangir Alam, M. Rabiul Alam, and Guoqing Hu
}

\begin{abstract}
In order to keep up with the increasing demand on mobile communications in everyday life including business and entertainment, the pressure to match the quality-of-service (QoS) level and performance of the high speed wired networks is paramount. Canopy antenna devices are supported in wireless network infrastructure and microwave link connectivity. The performances of canopy devices are depend on correlation of different parameters such as effect with others devices, links and systems, and with environmental effect, etc. This study is focused on the correlation of signal transmission and environmental parameters and how canopy devices can increase the performance in network connectivity.
\end{abstract}

Index Terms-Canopy, climate effect, traffic trends, correlations.

\section{INTRODUCTION}

Canopy antenna devices are supported in wireless network infrastructure and microwave link connectivity. Typical Canopy Subscriber Module operating frequencies are: 5.2, 5.7 or $2.4 \mathrm{GHz}$. The performances of canopy devices are depend on correlation of different parameters such as correlation effect with others devices, links and systems, correlation with environmental effect etc. To find out the problem faced by canopy devices, it has need to work about the environment in local area and have to know the specific areas characteristics of environmental effect, geographical information, rain effect, fog effect, attenuation, terrain effect, humidity, hi-voltage line effect, riverine vapor effect, water vapor, permittivity, permeability with refraction and reflection in the ground effect etc. Experimental investigations have shown to recover this problem. These were based on air properties changes with Temperature and Humidity, and the variation of parameters changes Permittivity with Temperature and Humidity. This study is focused on the correlation of signal transmission and environmental parameters and how the better uses of canopy devices can increase the canopy performance in network connectivity.

\section{Physical Transmission}

If there are hindrances between transmitter and receiver, the signals will weaken. A connection can thus become

Manuscript received January 17, 2014; revised March 13, 2014.

S. M. Jahangir Alam and Guoqing $\mathrm{Hu}$ are with the Department of Mechanical and Electrical Engineering, Xiamen University, China (e-mail: jahangir_uits@yahoo.com, gqhu@xmu.edu.cn).

M. Rabiul Alam is with the Department of Electrical and Electronic Engineering, Hamdard University Bangladesh, Narayangonj, Dhaka, Bangladesh (e-mail: alam2007@ mail.ru). problematic or impossible. In GSM there is therefore the possibility of regulation of the transmitting power (Power Control - PC) from mobile and base stations over several orders of magnitude [1], [2]. Multipath propagation through reflection and dispersion of radio waves leads to phase-shifted reception of signals of different paths. The interference can distort, amplify or erase the signal. An attempt to compensate for negative effects of multipath propagation is given by power control, frequency hopping, two antenna receivers for the base station (antenna diversity) and redundancy of the transmitted information [1], [2].

The distance between MS (Mobile Station) and BTS (Base Transreceiver Station) has proved to be problematic in several ways. The receive power sinks with increasing distance between transmitter and receiver theoretically with the square of the distance [3], [4]. Various physical effects such as atmospheric attenuation (weather-dependent) reduce the receive power even more. This attenuation depends on the frequency and increases with increasing frequency in mobile radio relevant frequency ranges. The distance furthermore causes a reception delay, which may lead to interference between neighboring time slots in TDMA (Time Division Multiplexing Access) [3], [5]. GSM (Global Systems for Mobile Communications) responds to this delay by means of a regulation of the transmission time (Timing Advance -TA). GSM900 cells (GSM Phase 1/2) are limited to maximum 35 $\mathrm{km}$, GSM1800 cells to maximum $8 \mathrm{~km}$ radius as a result of the distance related problems. There is the possibility in GSM Phase 2+ to realize "Extended Range Cells" with a maximum radius of $100 \mathrm{~km}$ for GSM900 [3], [6], [7].

Moving mobile stations can cause transmission distortions due to Doppler Effect. A compensation for this effect up to a maximum speed of $250 \mathrm{~km} / \mathrm{h}$, for GSM-R a more powerful compensation for speeds of up to $450 \mathrm{~km} / \mathrm{h}$ was developed. The receive quality can also be disturbed by electromagnetic waves from outside systems (e.g. car ignition, generators, PCs) [3], [6], [7].

\section{CANOPY NETWORK COMPONENTS AND CHARACTERISTICS}

Canopy Network Components. Canopy Broadband Wireless Components Consist of : Access Point Modules (AP) 5.0 GHz and 2.4 GHz, Subscriber Modules (SM) 5.0 GHz and $2.4 \mathrm{GHz}$, Backhaul Modules (BH) $5.0 \mathrm{GHz}$ and $2.4 \mathrm{GHz}$, Cluster Management Module (CMM), Surge Suppressor, Bandwidth and Authentication Manager (BAM), Simple Network Management Protocol (SNMP), Built-In and Third Party Management Mechanisms [8], [9].

Canopy Performance Characteristics. Access Points (AP's), Subscriber Modules (SM's) and 10Mb Backhaul Modules 
(BH) operates at a channel rate of $10 \mathrm{Mbps}(5.0 \mathrm{GHz}$ and 2.4 $\mathrm{GHz}$ ). 20Mb Backhaul Modules (BH) operate at a channel rate of $20 \mathrm{Mbps}(5.0 \mathrm{GHz}$ and $2.4 \mathrm{GHz})$. Modules operate in the U-NII and ISM bands [8], [9].

Canopy Radio Characteristics. Are available in the 5.2 $\mathrm{GHz}$ (U-NII), $5.7 \mathrm{GHz}$ (U-NII/ISM) and $2.4 \mathrm{GHz}$ (ISM) other bands determined by country [8], [9].

\section{DIFFERENT PARAMETERS AND CANOPY PERFORMANCE}

To find out the problem it have need to work about the environment in local area and have to know the specific areas characteristics of environmental effect, geographical information, rain effect, fog effect, attenuation, terrain effect, humidity, hi-voltage line effect, riverine vapor effect, water vapor, permittivity, permeability with refraction and reflection in the ground effect etc.

These studies were based on the air properties changes with Temperature and Humidity and the variation of parameters changes - Permittivity with Temperature and Humidity (Fig. 1-Fig. 5) of March 2006 for link performance of BanglaLink Mobile Communication (Orasscom Telecom Ltd.), have shown that when Humidity increases, propagation of wave takes extra time and attenuate power.

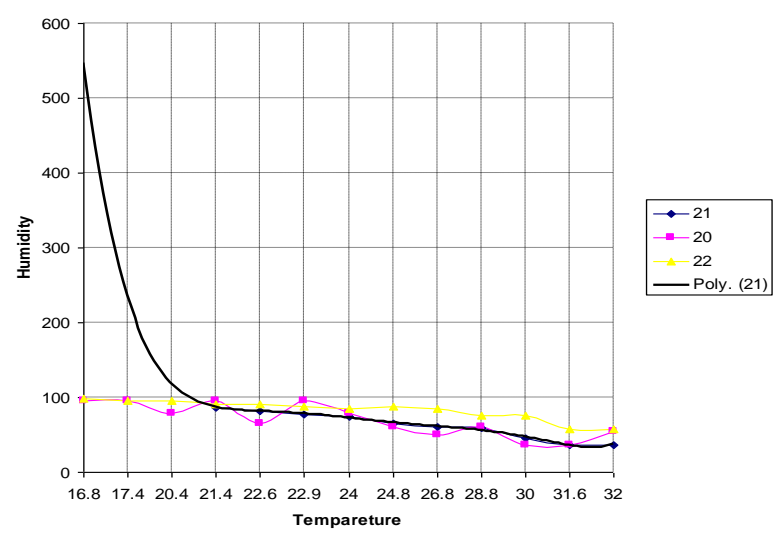

Fig. 1. Climate effect (temperature vs. humidity).

Temperature vs Permittivity

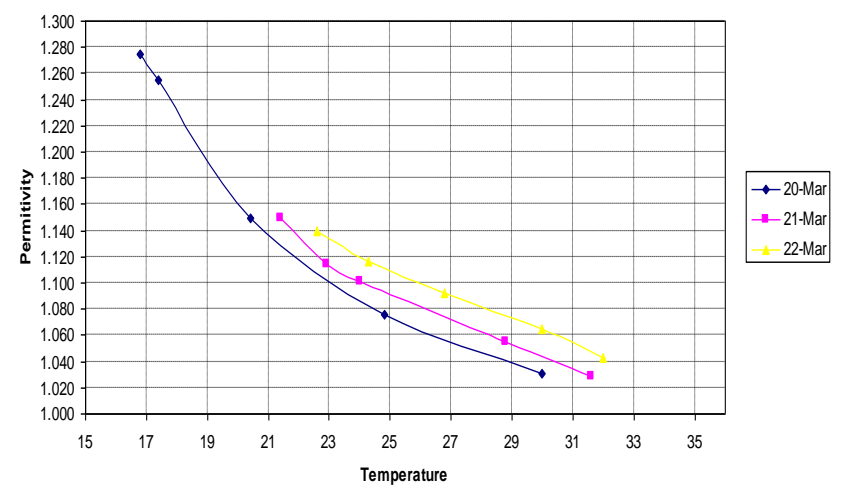

Fig. 2. Climate effect (temperature vs. permittivity).

All devices are set for certain time to receive a signal, when any signal fail to arrive within this time, receiver request for retransmission. This way it continue request for retransmission of signal until Humidity become normal. After sometime the whole systems become exhausted and fail to continue operate until the environment changes in normal condition. And as the environment became normal from $22^{\text {nd }}$
March and the link was started performing normally without any kind of device level changes. These studies have shown a correlation of Signal Transmission and Environment Parameters (Humidity and Temperature) [8], [10].

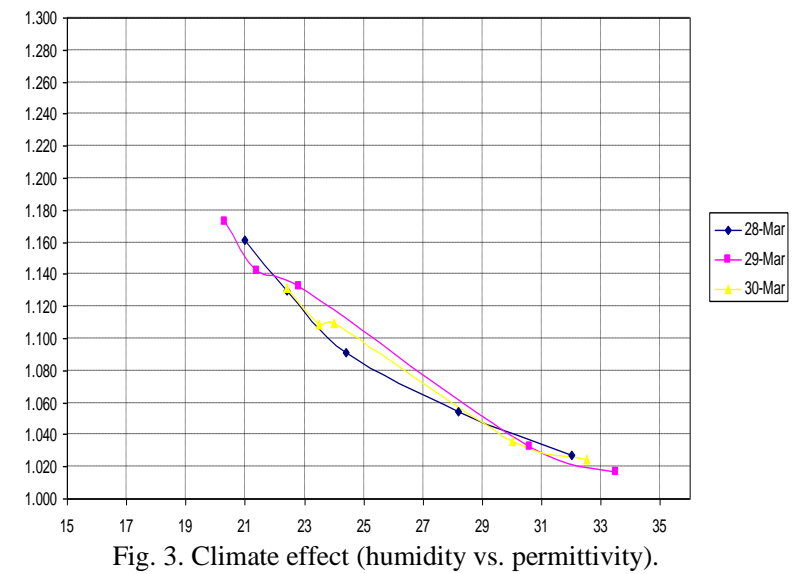

Humidity vs Permittivity

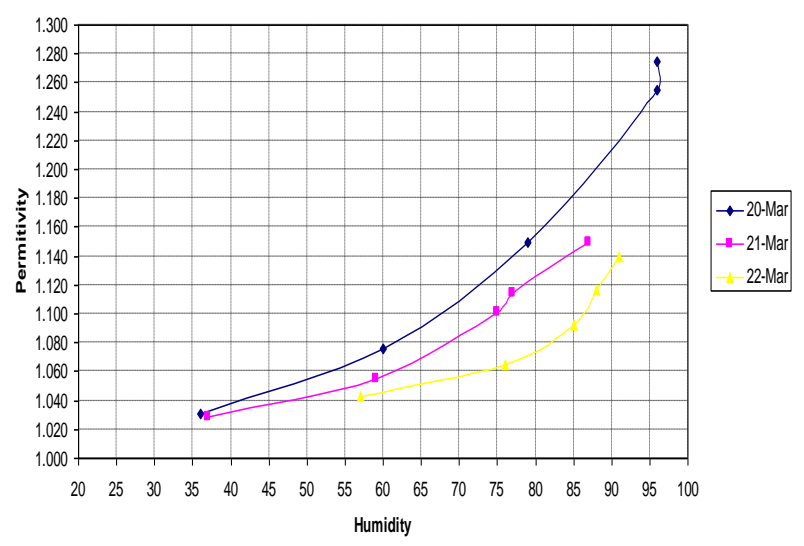

Fig. 4. Climate effect (humidity vs. permittivity).

Humidity vs Permitivity

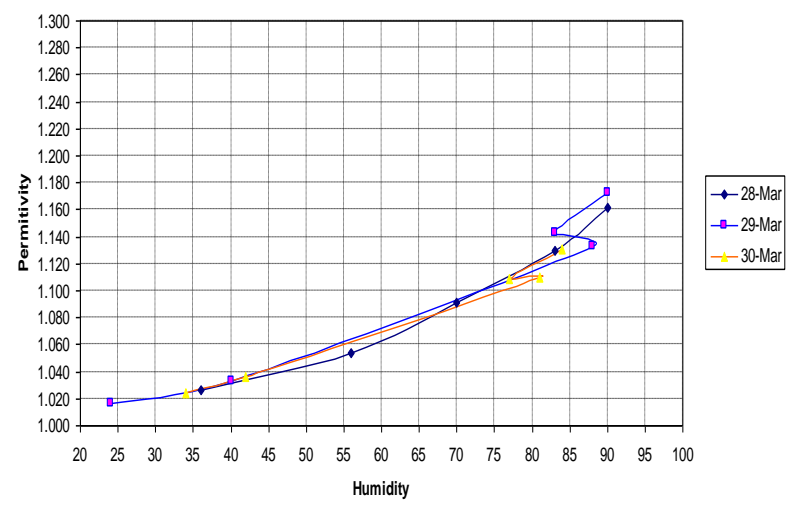

Fig. 5. Climate effect (humidity vs. permittivity).

\section{A. Attenuation}

Frequencies above $10 \mathrm{GHz}$ can be impacted by atmospheric conditions, weather and obstructions. Canopy performs well because it operates in the license exempt 5.0 $\mathrm{GHz}$ and $2.4 \mathrm{GHz}$ bands. Frequency can be affected by rainfall under certain conditions. Frequencies above $11 \mathrm{GHz}$ are dramatically affected by rainfall. When temperature increased rapidly then canopy are not accommodated the environment and call will be aborted, that means it QoS is not good [1], [8], [11]. 


\section{B. Climate Effects}

Impact of Weather and Atmospheric Conditions on 5.0 $\mathrm{GHz}$ and $2.4 \mathrm{GHz}$ Radios: Humidity - No Effect. But at 5.30 pm to $9.00 \mathrm{pm}$ the Humidity is increased rapidly (in figure 1), which was collected from the real data for March 2006 for BanglaLink Mobile Communication (Orasscom Telecom Ltd.), and if this variation is occurred, the call could have dropped [12].

\section{Rain Effect}

No Effect. But rapidly increasing the temperature canopy does not rapidly sense. If the environment is rainy, the device effects on frequency. That situation the permittivity and the frequency response are not sufficient on canopy performance. So when the canopy devices are installed on the mount that times an optimum RSSI value can use which can solve this problem. Whereas call will not be abort from GSM network and correlate with canopy device [10]. The rain effects are shown in the Fig. 6 and Fig. 7.

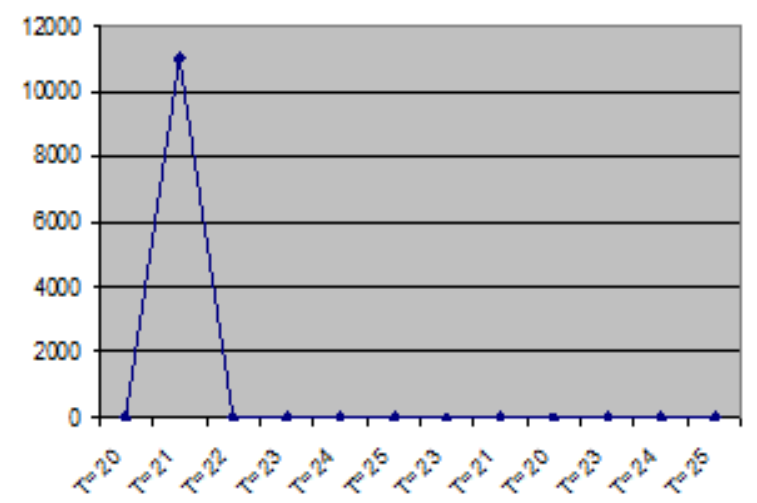

Fig. 6. Rain effect (time vs. distance).

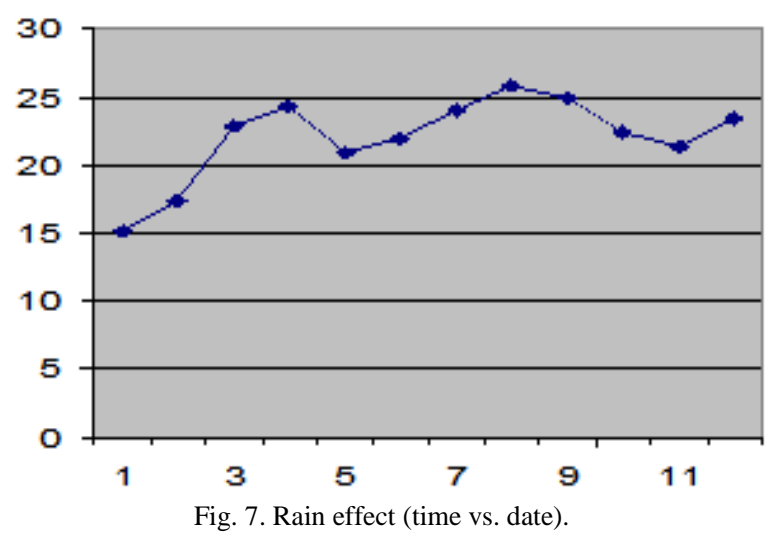

\section{Earth Curvature}

One factor to consider for line-of-sight microwave transmissions is earth curvature. Most path calculation software takes earth curvature into consideration. Earth curvature is not much of a factor under $16 \mathrm{~km}$ (10 miles). Over $16 \mathrm{~km}$ (10 miles) earth curvature must be added to site calculations [8].

\section{E. Earth Curvature}

One factor to consider for line-of-sight microwave transmissions is earth curvature. Most path calculation software takes earth curvature into consideration. Earth curvature is not much of a factor under $16 \mathrm{~km}$ (10 miles). Over
$16 \mathrm{~km}$ (10 miles) earth curvature must be added to site calculations [8].

\section{F. Fresnel Zone Clearance}

The first Fresnel Zone (pronounced Fresnel) is the locus of all points in space that result in a reflected ray $1 / 2$ wavelength out of phase at the intended receiver. The first Fresnel zone, when plotted in 3 dimensions, forms an ellipsoid. Simply stated - the signal is shaped like a three dimensional antennas). For best microwave performance, all obstructions should be kept out of the Fresnel Zone (Fresnel clearance). Most path calculations assume at least a $60 \%$ unobstructed Fresnel Zone clearance [4].

\section{G. Obstructions}

Some RF signals can easily travel through stone, trees, walls and other obstacles while other RF signals will be immediately attenuated. Partial obstructions can dramatically reduce the strength of an RF signal [4].

\section{H. Polarization}

Polarization is expressed as the plane in which radio waves propagate - Horizontal or vertical and circular. RF can be transmitted (and received) with a dominant polarization. Changing radio polarization will often permit two separate radio systems to co-exist in the same space. Weather and multipath can "de-polarize" RF [2].

\section{Radio Frequency Reflection}

Radio Frequency can bounce off of objects like buildings, mountains, bodies of water and the atmosphere. This causes the reflected and original signals to arrive at the receiver at different times - this is called 'multipath' [2].

\section{J. Terrain Effects}

Mountainous terrain is most desirable-Multipath is reduced because many reflections are cancelled and don't end up at the receiving end. Flat, smooth terrain is the least desirable. Many multipath reflections can end up at the receiving end, $1 \mathrm{~Hz}, 1 \mathrm{kHz}, 1 \mathrm{MHz}, 1 \mathrm{GHz}$ [10], [13]. The terrain effect is shown in the Fig. 8.

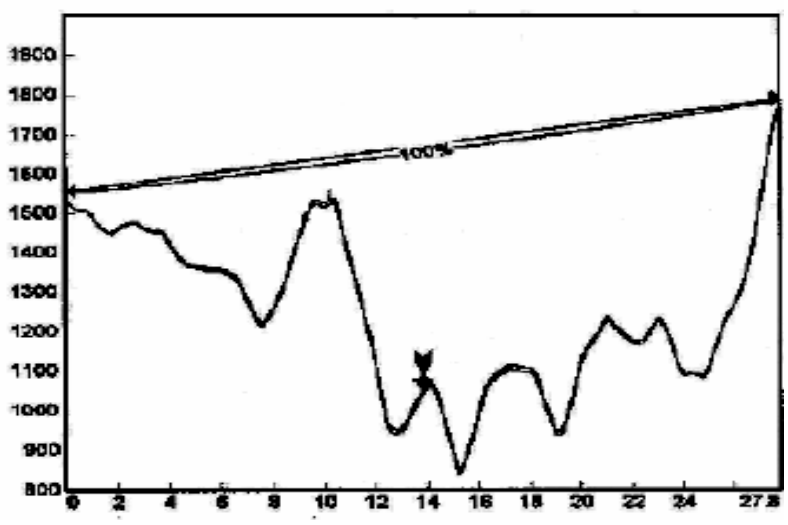

Fig. 8. Terrain effect

\section{K. Others Effect}

There are no other effects such as Snow effect, Ice effect, Wind effect. Humid climate is least desirable for microwave. More moisture-more attenuation. Dry climate is best for microwave. It is reduced moisture - less attenuation [10]. 


\section{CONCLUSION}

The performances of canopy devices are depend on correlation of different parameters such as correlation with others devices, links and systems, correlation with environmental effect etc. These studies were based on air properties changes with Temperature and Humidity, and the variation of parameters changes - Permittivity with Temperature and Humidity. When Humidity increases, propagation of wave takes extra time and attenuates power and the whole system become exhausted and fail to continue operate until the environment changes in normal condition. As the environment became normal and the link started performing normally without any kind of device level changes. A lot of research works is need in the direction on correlation of signal transmission and environmental parameters which can increase the performance of the canopy wireless devices in network connectivity.

\section{ACKNOWLEDGMENT}

It is sincere to gratitude and profound indebtedness to the support manager Mr. Md. Zaki and other experienced person of Ektoo.net. Without their inspiring and encouragement, this work could not have been completed. It is grateful to Dr. Hafizur Rahman of Bangladesh Abhawa Adhidaptor (Meteorological Dept.) for his contribution to complete this work by collecting data and for helping.

\section{REFERENCES}

[1] Z. Yun and M. F. Iskander, "Progress in modeling challenging propagation environments," in Proc. IEEE Antennas and Propagation Society International Symposium, 2004, vol. 4, pp. 3637-3640.

[2] M. D. Pozar, Microwave Engineering, 2nd ed.

[3] M. Tolstrup, Indoor Radio Planning: A Practical Guide for GSM, DCS, UMTS and HSPA, Chichester: Wiley, 2008.

[4] C. Y. W. Lee, Mobile Cellular Telecommunications, McGraw-Hill, 1995.

[5] K. Etemad, CDMA 2000, Evolution, System Concepts and Design Principles, John Wiley \& Sons, Inc., 2004.

[6] R. Horak, Telecommunications and Data Communications Handbook, John Wiley \& Sons, Inc., 2007.

[7] F. Simonds, Network Security: Data and Voice Communications, Series on Computer Communications, McGraw-Hill, 1995.

[8] Canopy Motorola Handbook for Wireless Communications, 2007.

[9] Cambium networks. [Online]. Available: http://motorola.canopywireless.com

[10] Practical Data, Banglalink Mobile Company, Bangladesh, 2006.

[11] W. Stalling, Data and Computer Communication, 2006.
[12] Bangladesh Abhawa Adhidaptor (Meteorological Department), Dhaka, Bangladesh.

[13] Prizmems Launched for Motorola's Canopy Wireless Networks, 2006.

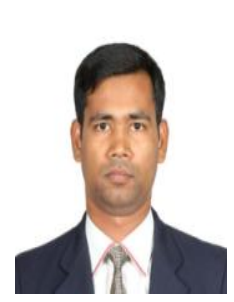

S. M. Jahangir Alam has completed B.Sc. in 2005 in computer science and engineering from Dhaka International University, Bangladesh and M.S. in Telecommunications from the Univ. of Info. Tech. \& Sci. (UITS), Dhaka, Bangladesh, 2008. He is a PhD candidate in the Dept. of Mechanical and Electrical Engineering at Xiamen University, China.

He was a lecturer of electronic and communication engineering and coordinator of the School of Comp. Sci. \& Engineering at UITS from 2006 to 2010 . He is interested in vision \& image processing, TFT-LCD display defect detection, time series analysis, detection technology, and automation.

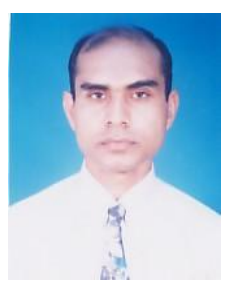

M. Rabiul Alam has completed M.S. in 1995 and awarded Ph.D. in electrical engineering from Moscow State Mining University, Moscow, Russia, 2004. From 2004 to 2006 he was a post-doctoral researcher in energy management at the same university.

$\mathrm{He}$ is an associate professor and the head of the Department of Electrical and Electronic Engineering at Hamdard University Bangladesh from 2012. During 2008-2012, he was an associate professor and head of the Dept of Electrical and Electronic Engineering and dean of the School of Comp. Sci. and Engineering at University of Information Technology and Sciences (UITS), Dhaka, Bangladesh. During 2007-2008 he was an associate professor of the Dept. of Electrical \& Electronic Engineering at International Univ. of Business Agriculture and Tech. (IUBAT), Dhaka, Bangladesh. His field of interests is: power engineering, renewable energy and energy management.

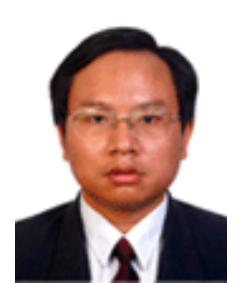

Guoqing Hu completed his B.S. in 1987 and M.S. in 1990 in the Dept. of Automation Control from Northwestern Polytechnical Univ. China and awarded $\mathrm{Ph} . \mathrm{D}$. in mechanical engineering from Sichuan Univ, China, 1993. In 1993-1995, he was a post-doctoral researcher in the Dept. of Mechanical Engineering, Shanghai Jiaotong University (PRC).

$\mathrm{He}$ is a professor of the Dept. of Mechanical \& Electrical Engineering at Xiamen University, China since 1995. During 1993-1995, he was an associate professor in the Dept. of Mechanical Engineering, Shanghai Jiaotong University. In Jan 2000-Feb 2001, he was a visiting professor in the Dept. of Electrical Engineering and Computer Science, Electrical Design Center, Case Western Reserve University (USA). From February 2002 to August 2002 he was a visiting professor in the Dept. of Automation and Computer-Aided Engineering at The Chinese University of Hong Kong (Hong Kong). His major research areas are: advanced sensors, electromechanical control, MEMS, high temperature measure and detection technology, industrial automation and industrial robot, fluid transmission and control, etc. 